\title{
Conception-why there is no rejection? - the lesson learned will revolutionize organ transplantation
}

\section{Editorial}

We know that if we try to transplant an organ from one person to another - the recipient will reject the organ. However, why is the future mother not rejecting the foreign sperms of the future father? Prof. Salvatore Mancuzo revealed the secret of this in his report at a congress organized by the Institute of Genecology at the Roman Catholic University in the fall of year 2000. Since the embryo will have features of the mother and the father, the future mother is forced to accept some features of the future child for the sperm to be accepted and not rejected. In this way the mother through the child is accepting some features of the father. ${ }^{1}$

According to the research of Prof. Mancuzo, after the $5^{\text {th }}$ week of pregnancy the future mother accepts from the embryo a bunch of changes - hormonal and stem cells changes. The embryo send his stem cells in the bone marrow of the mother, which colonize the mother's bone marrow and start producing their own lymphocytes. The embryo's lymphocytes remain in the mother's body for life. The embryo's own lymphocytes serve the purpose to avoid rejection - they are accepted as his mother's. Prof. Mancuzo answer to the question how long the mother's body keeps producing her child's lymphocytes was - they were able to detect them even 30 years after the birth of the child, but probably it continues for life.

Thus, not to reject the foreign (father's) sperm, the future mother needs to accept some changes in her body; these changes last for life and influence her body for life. A brilliant idea flashed through my mind - this finding means that when an organ is transplanted if we inject in the bone marrow of the receptor of the organ stem cells of the organ's donor, the organ will not be rejected. But there is another aspect of conception. Why so much sperm is needed for a conception to happen? In a fertilized cell the NEMF of father's DNA and the NEMF of mother's DNA will be present because everything material is a material body and nonlinear electromagnetic field (NEMF)). ${ }^{2}$ However, this is not enough. The fertilization is considered successful and the development of an embryo can start only if an additional informational nonlinear electromagnetic field (NEMF) is present.

This additional NEMF is what we see as aura - I measured its electrical component for almost 40 years. ${ }^{3-5}$ My measurements revealed that this NEMF is emotionally sensitive. Since we claim that we are in high spirit when we experience positive emotions (which makes our aura brighter), and we claim that we are in low spirit when we experience negative emotions (which makes our aura dimmer), we call this NEMF Spirit. Thus, all living beings (humans, animals, and plants) are a material body with its NEMF and magnetically attached to it is the emotionally sensitive Spirit (NEMF), which we see as aura.

I found that the Spirit is weak informational field (NEMF). If so, the Spirit's NEMF must have imprinted three-dimensional holographic image of the individual to be and the three-dimensional embryo develops under its rule. According to my measurements, later this weak informational field rules and regulates everything in the material body of the individual not with its strength, but with the information it carries. The NEMF, called Spirit, makes all living being (humans, animals, and plants) emotionally sensitive and allows the
Volume 9 Issue 4 - 2020

\author{
Maria Kuman \\ Holistic Research Institute, TN 37923, USA
}

Correspondence: Maria Kuman, Holistic Research Institute, I 4 I 4 Barcelona Dr., Knoxville, TN 37923, USA, Email holisticre@mariakuman.com

Received: November 03, 2020 | Published: November 23, 2020

functioning of their organs to be modulated by emotions. In ancient Chinese sources: 1/ the upper part of he body is Yang, which means active; 2/ the lower part of the body is Yin which means passive.

The material body is passive (Yin) and what activates it (Yang) (by bringing life and emotions to it) is the Spirit (Yang). Since the Spirit (seen as aura) is nonlinear (NEMF) and all nonlinear fields exhibit turbulence manifested as vortices and anti-vortices, the human NEMF has a chain of 7 alternating vortices (spinning clockwise and sucking energy in) and anti-vortices (spinning counterclockwise and emitting energy out). They are along the backbone and they are called "chakras", which means "spinning wheels" in Sanskrit.

Thus, our Spirit (NEMF) is a living NEMF entity breathing energy in through its vortices and breathing energy out through its antivortices. Chakra \#1 at the tailbone is open to the Earth and connects the Spirit (NEMF) to the magnetic field (NEMF) of the Earth; chakra \#7 on top of the head is open to the atmosphere and connects the Spirit (NEMF) to the electric field (NEMF) of the atmosphere. The rest of the chakras are open in front and back of the body and connect the Spirit (NEMF) to the environment.

Since the Spirit is NEMF, the energy it breathes in from the environment must be NEMF energy, which means that NEMF is around us. This is the primary substance from which everything was created. I call it "Space Matrix", but it was called "ether" in the past. Thus the ether is NEMF. This explains why we denied the existence of ether for so long - NEMF is invisible and NEMF is difficult to measure because it is a weak informational field. It is 1,000 times weaker than the EMF, which our nervous system creates. I had to develop a special very sensitive equipment to be able to measure it.

In my article,${ }^{6}$ I explained that the passive (Yin) part is in the lower material body (Yin). The body has two Yin chakras: chakras \#1 at the tailbone, called "Earth Chakra" because it is open to the Earth and connects us to the NEMF of the Earth, and chakra \#2 under the belly button called Sexual Chakra. To be attracted to the opposite sex, the sexual chakras of the opposite genders spin in opposite direction and the whole NEMFs of individuals with opposite sex spin in opposite direction at the same time of day or night. The two chakras (\#1 and \#2) are the vortex and anti-vortex of the NEMF of the material body.

In my article,${ }^{6}$ I explained that the upper part of the body is Yang because the active (Yang) Spirit operates there. The Spirit (Yang) has two Yang chakras: chakras \#5 on the throat called "Throat Chakra" 
and chakra \#6 on the forehead called "Chakra of Intuition". These are the vortex and anti-vortex of the NEMF of the Spirit. The middle two chakras: chakra \#3 called "Chakra of the Solar Plexus" and chakra \#4 called "the Heart Chakra" belong to both - the material body and the Spirit. We call them "Chakras of the Soul".

Thus, Soul by definition is the unity of body and Spirit. ${ }^{6}$ For this reason, we consider the Heart the seat of the Soul and the two chakras (\#3 and \#4) of the Soul are the vortex and anti-vortex of the NEMF of the Soul, which is sum of the NEMF of the Spirit and NEMF of the material body). So, in all living beings we have the NEMF of the material body intertwined with the NEMF of the emotional Spirit. To attract each other magnetically, the two NEMFs must spin in opposite direction and they do.

Kundalini Yoga speaks about Kundalini energy running along the backbone as two intertwined spirals - one running downward (which is the spiral NEMF energy of the Spirit) and the other running upward (which is the spiral NEMF of the material body). ${ }^{7}$ Since during the day the NEMFs (auras) of men (Yang) spins clockwise like a vortex and suck electrical (Yang) energy from the atmosphere, ${ }^{6}$ for men dominant will be the Kundalini spiral running downward. Since during the day the NEMFs (auras) of women (Yin) spin counterclockwise like an anti-vortex and suck energy from the Earth (Yin), for women dominant will be the Kundalini spiral running upward.

But let us go back and see what happens when a cell is considered successfully fertilized. The first thing we see is a beating heart. The fact that the heart is the first to start functioning in an embryo is a proof that we are a material body and Spirit because the Heart Chakra is Chakra of the Soul and Soul is the unity of body and Spirit.

\section{Acknowledgments}

None.

\section{Conflicts of interest}

The authors declare that they have no conflicts of interest.

\section{Funding}

None.

\section{References}

1. V Tihoplav, T Tihoplav. The Harmony of the Chaos. 2003. Russia;

2. M Kuman. How the Material World Was Created? - Origin of Its NEMF. Open Acc J Math Theor Phy. 2019;2(2):34-38.

3. M Kuman. The Keys to Health and Happiness - Measurements Show that It is Not only Important what You Eat and Drink, It Is Equally Important what You Think. Current Trends in Bioengineering and Biosciences. 2019;18(1).

4. M Kuman. Pandemic and the Detrimental Effect of Negative Thinking and Emotions (Especially Fear) on Our Immunity. Global Journal of Science Frontier Research. 2009;20(5).

5. M Kuman. Our Weak NEMF that Rules Everything Is Emotionally Sensitive. Journal of Complimentary Medicine and Alternative Healthcare. 2018;8(2).

6. M Kuman. Health and Happiness Means Balance. EC Emergency Medicine and Critical Care. 2019;4(4).

7. M Kuman. Yoga - Health Benefits, Wisdom, and Science. Health and Happiness Books. 1999 